\title{
Internetbasierte Therapie nach Verlust eines Kindes in der Schwangerschaft - Einfluss sozialer Unterstützung auf die Verarbeitung des Verlusts
}

\author{
Grit Klinitzke Ruth Dölemeyer* Jana Steinig Birgit Wagner Anette Kersting \\ Klinik und Poliklinik für Psychosomatische Medizin und Psychotherapie, Universitätsklinikum Leipzig AöR, Deutschland
}

\section{Schlüsselwörter \\ Internetbasierte Psychotherapie · Pränatalverlust . \\ Soziale Unterstützung · Schreibtherapie}

\section{Zusammenfassung}

Hintergrund: Der Verlust eines Kindes in der Schwangerschaft stellt für die betroffenen Eltern eine hohe psychische Belastung dar, die zu manifesten psychischen Erkrankungen führen kann. Verschiedene Studien haben gezeigt, dass die wahrgenommene soziale Unterstützung durch das Umfeld der Betroffenen nach einem pränatalen Kindsverlust mit geringerer prolongierter Trauersymptomatik einhergeht. Methode: Untersucht wurden 99 Eltern, die ihr Kind während der Schwangerschaft verloren hatten. Sie nahmen an einem 5-wöchigen internetbasierten Behandlungsprogramm zur Schreibtherapie teil, welches die 3 Phasen "Selbstkonfrontation", "kognitive Umstrukturierung" und "Social Sharing" beinhaltete. Neben allgemeiner psychischer Belastung (BSI), prolongierter Trauer (ICG) und traumatischem Stress (IES-R) wurde auch die soziale Unterstützung (BSSS) mit den Dimensionen "wahrgenommene Unterstützung" und "erhaltene Unterstützung" bzw. "Bedürfnis nach Unterstützung" und "Suche nach Unterstützung" sowohl zum Zeitpunkt vor der Behandlung als auch nach deren Abschluss erhoben. Ergebnisse: Während das Bedürfnis nach sozialer Unterstützung im Verlauf der Behandlung signifikant abnahm, erhöhten sich die Werte der anderen 3 Dimensionen sozialer Unterstützung signifikant. Die wahrgenommene und erhaltene soziale Unterstützung waren signifikant invers mit der allgemeinen psychischen Belastung und der prolongierten Trauer nach einem pränatalen Verlust assoziiert. Erhaltene Unterstützung und die Suche nach Unterstützung gingen mit der Verbesserung verschiedener Symptome nach der internetbasierten Behandlung einher. Schlussfolgerung: Die Ergebnisse weisen darauf hin, dass sowohl die erhaltene Unterstützung als auch die aktive Suche nach Unterstützung für die Wirksamkeit des internetbasierten Behandlungsprogramms für Betroffene nach einem Kindsverlust relevant sind. Zudem kann das hier vorgestellte internetbasierte Behandlungsangebot nach dem pränatalen Verlust eines Kindes eine gute Unterstützungsmöglichkeit darstellen und aktivierend bei der Suche nach Unterstützung wirken.

*R.D. trug gleichermaßen als Erstautorin zu dieser Publikation bei

\author{
Keywords \\ Internet-based psychotherapy · Prenatal child loss . \\ Social support - Writing therapy

\section{Summary} \\ Internet-Based Therapy after Pregnancy Loss - the Impact of \\ Social Support on Bereavement
}

Background: The loss of a child during pregnancy poses a severe psychological strain for the parents concerned and can lead to manifest mental illness. Various studies have shown that perceived social support by the environment of those affected by a prenatal child loss goes along with less prolonged grief symptoms. Method: We investigated 99 parents who had lost their child during pregnancy. They took part in a 5 -week internet-based treatment program for writing therapy, which included the 3 phases "confrontation", "cognitive restructuring", and "social sharing". In addition to general psychological distress (BSI), prolonged grief (ICG), and traumatic stress (IES-R) social support (BSSS) was evaluated with the dimensions "perceived support" and "received support" and "need for support» and "seeking support» both at the time before and after treatment. Results: While the need for social support significantly decreased in the course of treatment, the values of the other 3 dimensions of social support increased significantly. The perceived and the received social support were significantly inversely associated with general psychological distress and prolonged grief after a prenatal loss. Received support and seeking support were associated with the improvement of several symptoms after the internet-based treatment. Conclusion: The results indicate that both the received support and actively seeking support are relevant for the effectiveness of the internet-based treatment program for those affected by the loss of a child. Moreover, the internet-based treatment program presented here can offer a good support option after prenatal loss of a child and contribute to activation of seeking support.

\begin{tabular}{ll}
\hline KARGER & @ 2013 S. Karger GmbH, Freiburg \\
Fax +49 7614520714-6262/13/0233-0181\$38.00/0 \\
$\begin{array}{l}\text { Information@Karger.com } \\
\text { www.karger.com }\end{array}$ & $\begin{array}{l}\text { Accessible online at: } \\
\text { www.karger.com/ver }\end{array}$
\end{tabular}

Dr. Dipl.-Psych. Grit Klinitzke Department für Psychische Gesundheit Klinik und Poliklinik für Psychosomatische Medizin und Psychotherapie Universitätsklinikum Leipzig AöR Semmelweisstraße 10, 04103 Leipzig, Deutschland grit.klinitzke@medizin.uni-leipzig.de 


\section{Einleitung}

Der Verlust eines Kindes während der Schwangerschaft nimmt in der Öffentlichkeit trotz hoher Prävalenz noch immer wenig Raum ein. So wird die Prävalenzrate einer Fehlgeburt für alle Schwangerschaften auf bis zu 50\% geschätzt, wobei ca. $15 \%$ aller klinisch diagnostizierten Schwangerschaften in einer Fehlgeburt enden [Gold et al., 2007; Geller et al., 2010]; Totgeburten treten bei ca. 1\% aller Geburten auf [Gold et al., 2007]. Ein solcher Verlust des ungeborenen Kindes stellt für viele der Betroffenen eine massive psychische Belastung dar [Berth et al., 2009], die bei den Eltern zu langanhaltenden Trauerprozessen und manifesten psychischen Erkrankungen führen kann [Brier, 2004, 2008; Kersting, 2012; Kersting und Wagner, 2012]. Weiterhin konnte in verschiedenen Studien gezeigt werden, dass der Verlust eines Kindes während der Schwangerschaft auch für nachfolgende Schwangerschaften gravierende Auswirkungen haben kann. So wurde in einer prospektiven Studie [Bergner et al., 2008] deutlich, dass eine frühe Fehlgeburt mit erhöhter Angst und erhöhter depressiver Symptomatik in der nächsten Schwangerschaft einhergehen kann. Weiterhin kann die Bindung der Eltern an ein nachfolgend geborenes Kind beeinträchtigt werden [Kersting und Wagner, 2012]. Neben einer bereits bestehenden psychischen Erkrankung, Kinderlosigkeit, einer belasteten Partnerbeziehung, einer früheren Fehlgeburt und einer ambivalenten Beziehung zum ungeborenen Kind kann auch ein geringes $\mathrm{Ma}$ an sozialer Unterstützung als ein relevanter Risikofaktor für die Entwicklung einer schweren und andauernden psychischen Belastung nach einer Fehlgeburt gesehen werden [Janssen et al., 1997; Lok und Neugebauer, 2007; Kersting und Wagner, 2012].

Sowohl unterstützende Einzel- oder Gruppenangebote zum Erfahrungsaustausch und zum Erlernen von Bewältigungsstrategien [Forrest et al., 1982; Cuisinier et al., 1993; Janssen et al., 1997; Cote-Arsenault und Freije, 2004; Sutan und Miskam, 2012] als auch psychotherapeutische Interventionen können einen positiven Einfluss auf das Verarbeiten eines Kindsverlusts haben und vor der Entwicklung psychopathologischer Symptome wie z.B. prolongierter Trauersymptome schützen [Neugebauer et al., 2006; Swanson et al., 2009; Kersting und Wagner, 2012]. Auch ein internetbasiertes Behandlungsangebot für trauernde Eltern nach pränatalem Verlust konnte allgemeine psychische Belastung, traumatisches Erleben und prolongierte Trauersymptome reduzieren [Kersting et al., 2011a; 2013]. Häufig besteht in der Öffentlichkeit Unverständnis über eine potenzielle emotionale Belastung, die durch eine frühe Fehlgeburt ausgelöst werden und langfristig bestehen kann. Zudem mangelt es oftmals an Informationen zum Umgang mit einer solchen Situation, die die Bewältigung einer frühen Fehlgeburt für Paare erleichtern könnten. Dies führt bei vielen Frauen dazu, über das eigene Erleben nur mit dem Partner oder engen Freunden und nicht mit anderen Personen zu sprechen [Conway, 1995]. Weiterhin können vor allem Frauen z.B. aufgrund eines ambivalenten Gefühls zur oder Risikoverhaltens während der Schwangerschaft durch mögliche Schuld- und Schamgefühle zusätzlich belastet sein [Leppert and Pahlka, 1984; Janssen et al., 1997]. Diese Gründe können zu einem sozialen Rückzug der Betroffenen führen und erfordern dadurch besondere Hilfsangebote wie beispielsweise internetbasierte Behandlungsverfahren.

Neben professioneller Unterstützung stellt vor allem die soziale Unterstützung im privaten Bereich einen wichtigen Aspekt im Umgang mit einem Kindsverlust während der Schwangerschaft dar. So wird die Entstehung psychopathologischer Belastung im Allgemeinen und prolongierter Trauer im Besonderen unter anderem auf mangelnde soziale Unterstützung durch den Partner und durch Freunde zurückgeführt [Forrest et al., 1982; Kuse-Isingschulte et al., 1996; Lok und Neugebauer, 2007; Kersting et al., 2011b; Sutan und Miskam, 2012]. Dabei wird ein Verlust als stressintensiv auf der affektiven Ebene empfunden; dies kann bei mangelnden individuellen Ressourcen die Intensität negativer Emotionen wie Trauer, Angst oder Ärger verstärken [Hobfoll, 1989]. Nach dem Haupteffektmodell der sozialen Unterstützung hat die bereits vorhandene Unterstützung eine positive Wirkung auf das Wohlbefinden und kommt nicht nur dann zum Tragen, wenn starker Stress wie z.B. durch den pränatalen Verlust eines Kindes vorliegt [Park et al., 2004; Kienle et al., 2006]. Dies geht mit den Erwartungen des Unterstützungsempfängers einher, ob und wie viel Unterstützung potenziell in belastenden Situationen verfügbar ist und was als wahrgenommene soziale Unterstützung bezeichnet wird [Kienle et al., 2006]. Viele Untersuchungen zeigen, dass hohe Werte wahrgenommener sozialer Unterstützung mit geringer Trauer bei Verlust eines Kindes in der Schwangerschaft einhergehen [Lasker und Toedter, 1991; Toedter et al., 2001; Sutan and Miskam, 2012]. Ergänzend ist die Assoziation zwischen wenig intimer oder stützender Partnerschaft und intensiven Trauergefühlen nach einem Kindsverlust beschrieben worden [Lasker und Toedter, 1991; Cuisinier et al., 1993; Bergner et al., 2009; Carlbring et al., 2012]. Von der wahrgenommenen sozialen Unterstützung abzugrenzen ist die tatsächlich erhaltene soziale Unterstützung, der im sogenannten Puffermodell ein abschirmender Effekt zugesprochen wird und die so während des Stressprozesses vor psychischer Belastung schützt [Klauer und Schwarzer, 2001; Kienle et al., 2006].

Die soziale Unterstützung selbst wurde in Studien zum Kindsverlust nur selten differenziert und standardisiert erhoben. Allerdings können gemäß eines mehrdimensionalen Ansatzes neben wahrgenommener und erhaltener Unterstützung auch das Bedürfnis und die Suche nach sozialer Unterstützung unterschieden und standardisiert erhoben werden [Schulz und Schwarzer, 2003]. Aus diesem Grund wurde in der vorliegenden Studie untersucht, inwiefern die wahrgenommene und erhaltene soziale Unterstützung der Betroffenen sowie deren Bedürfnis und die Suche nach sozialer Unterstützung 1) sich nach Abschluss eines internetbasierten 
Behandlungsprogramms nach pränatalem Kindsverlust verändern, 2) im Zusammenhang mit Aspekten der psychischen Belastung (traumatisches Erleben, prolongierte Trauer und allgemeine psychische Belastung) durch den Verlust stehen, und 3) ob die soziale Unterstützung mit ihren verschiedenen Dimensionen mit dem Behandlungseffekt einer internetbasierten Intervention korreliert.

\section{Methode}

\section{Studienteilnehmer}

Müttern und Vätern, die während der Schwangerschaft ein Kind verloren hatten, wurde zur Bewältigung der Trauer ein internetbasiertes Behandlungsprogramm im Rahmen eines Forschungsprojekts angeboten. Das Angebot der internetbasierten Intervention wurde über verschiedene Medien wie Radio, Fernsehen und Zeitungsanzeigen beworben. Zusätzlich wurden niedergelassene Gynäkologen und Kliniken angeschrieben und gebeten, die Informationen über das Behandlungsangebot an betroffene Eltern weiterzugeben. Bundesweite Selbsthilfegruppen wurden angeschrieben mit der Bitte, auf das Behandlungsprogramm in persönlichen Kontakten bzw. auf ihrer Webseite zu verweisen. Diejenigen, die sich für eine Teilnahme an der Intervention interessierten, konn ten auf der Internetseite, über die die Behandlung angeboten wurde (www.internettherapie-trauernde-eltern.de), weitere Informationen über das Angebot einholen oder sich für die Teilnahme anmelden. Um an der Studie teilnehmen zu können, mussten folgende Einschlusskriterien erfüllt sein: 1) der Verlust eines Kindes während der Schwangerschaft durch eine Fehlgeburt, eine Totgeburt oder einen medizinisch indizierten Schwangerschaftsabbruch; 2) wohnhaft in einem deutschsprachigen europäischen Land; 3) Fähigkeit, fließend Deutsch zu lesen und zu schreiben; 4) regelmäßiger Zugang zum Internet und 5) schriftliche Zustimmung zur Studienteilnahme. Ausschlusskriterien waren: 1) Minderjährigkeit; 2) eine aktuell bestehende Schwangerschaft; 3) eine aktuell laufende psychotherapeutische Behandlung und 4) hohe psychische Belastung wie starke depressive Symptomatik, suizidale Tendenzen, hohe Dissoziation, psychotische Symptome, Substanzmissbrauch oder -abhängigkeit. Diese Symptome wurden mit Hilfe eines Sets von Screening-Fragebögen online erhoben. Interessenten, die eine hohe psychische Belastung zeigten, wurden telefonisch kontaktiert und bei Bestätigung der vermuteten Symptome aus der Studie ausgeschlossen. In diesen Fällen wurden Informationen über mögliche Behandlungsalternativen zur Verfügung gestellt und die Interessenten - wenn gewünscht - bei der Suche nach einem Therapeuten unterstützt.

Nach Einschluss in die Studie wurden die Teilnehmenden entsprechend des Wartegruppen-Kontrolldesigns randomisiert, d.h. die Personen wurden entweder der Behandlungsgruppe (BG), die sofort mit der Intervention beginnen konnte oder der Wartelistengruppe (WL), die erst nach 5 Wochen Wartezeit mit der Behandlung begann, zufällig zugeordnet. Die Randomisierung wurde mit Hilfe eines Online-Randomisierungsservices (www.random.org) von der Studienkoordinatorin durchgeführt. Dabei wurden die Charakteristika der Studienteilnehmer nicht stratifiziert. Die Durchführung der Studie wurde gemäß der ethischen Richtlinien der Ethikkommission des Universitätsklinikums Münster akzeptiert.

\section{Intervention}

Sowohl die Intervention als auch die Wartezeit dauerten jeweils 5 Wochen. Die Intervention basierte auf einer Weiterentwicklung eines kognitiv-verhaltenstherapeutischen Ansatzes (KVT) und einer Schreibtherapie für Posttraumatische Belastungsstörung (PTBS), die von Lange und Kol- legen [Lange et al., 2003] entwickelt wurde. Bei dieser Form der Schreibtherapie kommunizieren Therapeut und Patient ausschließlich textbasiert und zeitlich asynchron per E-Mail. Die Intervention besteht aus strukturierten Schreibaufgaben, die von Pennebaker und Kollegen (wie von McLellan und Kollegen [1981] beschrieben) konzipiert wurden. Auf der Grundlage dieses Behandlungsmanuals entwickelten Wagner und Kollegen [2006] eine internetbasierte Intervention für prolongierte Trauer, die für die vorliegende Studie für trauernde Eltern nach pränatalem Verlust übernommen wurde. Die Intervention beinhaltete 10 Schreibaufgaben, die in die 3 Phasen «Selbstkonfrontation», «kognitive Umstrukturierung» und «Social Sharing» gegliedert waren. Während die ersten beiden Behandlungsphasen jeweils das Schreiben von 4 Texten beinhalteten, wurden in der letzten Phase nur 2 Texte geschrieben. Nach jedem zweiten Text bekamen die Teilnehmenden eine individuelle Rückmeldung und eine neue Schreibanleitung innerhalb von 2 Werktagen durch ihre persönliche Therapeutin. Nur bei den letzten beiden Schreibaufgaben, die sich auf das Social Sharing bezogen, bekamen sie schon nach dem ersten Text eine ausführliche Rückmeldung und eine Schreibanleitung für das Verfassen des letzten Textes. Eine detaillierte Beschreibung des Behandlungsprogramms und der Effektivität der Behandlung ist bei Kersting und Kollegen zu finden [Kersting et al., 2011a,c; 2013]. Die Rückmeldezeit der Therapeutinnen variierte zwischen 20 und 50 min pro Text, in Abhängigkeit von deren Erfahrung mit internetbasierten Verfahren und von der Länge der Teilnehmertexte. Mit Ausnahme einer approbierten Kraft befanden sich alle Therapeutinnen in entsprechender Weiterbildung zur Psychologischen Psychotherapeutin, waren in KVT geschult und erhielten wöchentlich Supervision.

\section{Messinstrumente}

Alle eingesetzten Erhebungsinstrumente waren Selbstbeurteilungsfragebögen, die vor Behandlungsbeginn (Baseline), am Ende der Behandlung (Post-Messung) und zu den 2 Follow-up-Zeitpunkten (3 und 12 Monate nach Abschluss der Behandlung) erhoben wurden. Zusätzlich wurden zum Zeitpunkt der Baseline noch weitere relevante Aspekte wie Alter, Beziehungsstatus, Schwangerschaftswoche, in der der Verlust stattfand, und die Art des Verlusts (frühe Fehlgeburt, späte Fehlgeburt, Totgeburt oder medizinisch indizierter Abbruch) erfasst. Weiterhin wurde erfragt, wie lange der Verlust zurücklag und wie eventuelle frühere Schwangerschaften verliefen. Ergebnisse hinsichtlich traumatischer Belastung, prolongierter Trauer und allgemeiner Psychopathologie wurden wie folgt erfasst.

\section{Traumatische Belastung}

Die traumatische Belastung wurde mit der deutschen Version der Impact of Event Scale-Revised (IES-R, deutsche Version [Maercker und Schützwohl, 1998]) erhoben. Dieser Fragebogen umfasst die 3 Subskalen «Intrusion», «Vermeidung» und «Übererregung». Diese Subskalen bestehen aus insgesamt 22 Items, die die Häufigkeit der einzelnen Symptome in den letzten 7 Tagen auf einer 4-stufigen Likert-Skala ( 0 = gar nicht; $1=$ selten; $3=$ manchmal; 5 = oft) erfassen. In der vorliegenden Untersuchung wurden die Teilnehmenden gebeten, die Fragen der IES-R auf den Verlust ihres Kindes zu beziehen.

\section{Prolongierte Trauer}

Das Ausmaß der prolongierten Trauer wurde mit Hilfe der deutschen Version des Inventory of Complicated Grief (ICG, deutsche Version Rosner, 2002, persönliche Mitteilung) gemessen. Dieser Fragebogen erfasst eine große Spannbreite von Kognitionen, Emotionen und Verhaltensweisen, die mit einer Trauerreaktion verbunden sind, wie sie von Prigerson und Kollegen [Prigerson et al., 1995] beschrieben wird. Der Fragebogen besteht aus 15 Items, die durch die Teilnehmenden auf einer 5-stufigen Likert-Skala (1-5) beantwortet werden sollten. 


\section{Allgemeine Psychopathologie}

Zur Erfassung der allgemeinen Psychopathologie wurde die deutsche Version des Brief Symptom Inventory (BSI, deutsche Version [Franke, 2000]) herangezogen. Sie umfasst 53 Items, die sich auf die physischen und psychischen Symptome der letzten 7 Tage beziehen und durch die Teilnehmenden auf einer 4-stufigen Likert-Skala eingeschätzt werden sollten. Der BSI beinhaltet neben einer allgemeinen Skala zur mentalen Gesundheit (Global Severity Index, GSI) verschiedene Subskalen wie die hier untersuchten Skalen «Depressivität» und «Ängstlichkeit».

\section{Soziale Unterstützung}

Zur Erfassung der sozialen Unterstützung wurden die Berliner Social Support Skalen (BSSS) [Schulz und Schwarzer, 2003] eingesetzt, die für die mehrdimensionale Erfassung sozialer Unterstützung entwickelt wurden. Die BSSS erfassen auf 34 Items die 5 Dimensionen 1) «wahrgenommene soziale Unterstützung», 2) «erhaltene soziale Unterstützung», 3) «Bedürfnis nach sozialer Unterstützung», 4) «Suche nach sozialer Unterstützung» und 5) «protektives Abfedern» aus der Perspektive von Patienten und Betroffenen. Von diesen 5 Dimensionen wurden in der vorliegen den Studie die ersten 4 erfasst. Die Antwortskala enthält 4 Stufen, die die Zustimmung von 1 («Ich stimme überhaupt nicht zu») bis 4 («Ich stimme stark zu») erfassen.

Die Dimension «wahrgenommene soziale Unterstützung» wurde mit 8 Items erfasst, bezog sich auf das vom Betroffenen wahrgenommene Unterstützungspotenzial des sozialen Netzwerks und wurde als zeitlich unabhängiges Konstrukt konzipiert. Im Gegensatz dazu wurde die Dimension «erhaltene soziale Unterstützung», die mit 11 Items erfasst wurde, retrospektiv und mit Bezug auf die spezielle Situation und eine enge Bezugsperson konzipiert. Die 3. Dimension, das «Bedürfnis nach sozialer Unterstützung», bezieht sich sowohl auf Situationscharakteristika als auch auf situationsübergreifende persönliche Präferenzen in Bezug auf soziale Unterstützung. Zur Erfassung dieser Dimension werden 4 Items eingesetzt. Die «Suche nach sozialer Unterstützung» beschreibt ein aktives Bemühen, das soziale Netzwerk zu mobilisieren, um Stress bewältigen zu können. Aus diesem Grunde kann diese Dimension, die mit 5 Items erfasst wird, der Bewältigung zugeordnet werden. Auch hier wurden die Mobilisierungsbemühungen situationsspezifisch erfragt.

\section{Statistische Analysen}

Alle statistischen Analysen wurden mit der Software SPSS (Version 20.0) durchgeführt. Zur Berechnung der sozialen Unterstützung wurden die Mittelwerte der BSSS-Subskalen berechnet und die Unterschiede zwischen den beiden Messzeitpunkten mittels t-Tests (abhängige Stichproben) überprüft. Weiterhin wurden hier die Effektstärken nach Cohens d berechnet [Cohen, 1988]. Zur Erfassung des Zusammenhangs zwischen der sozialen Unterstützung und der Symptomatik bzw. dem Behandlungseffekt wurden die Korrelationen der BSSS mit den Prä- und PostWerten des ICG, des IES-R und der Skalen «Depressivität» und «Ängstlichkeit» sowie des GSI des BSI bzw. den entsprechenden Residual Gain Scores (RGS) berechnet. Der RGS berücksichtigt anfängliche Unterschiede und potenzielle Messfehler [Steketee and Chambless, 1992]. Die Berechnungsformel des RGS ist $\mathrm{z} 2-\left(\mathrm{z} 1 * r_{12}\right)$, wobei z2 und z1 die z-transformierten Werte zur Post- bzw. Prä-Messung darstellen und $r_{12}$ die Korrelation dieser beiden Werte repräsentiert.

\section{Ergebnisse}

\section{Studienteilnehmer}

Zwischen März 2009 und September 2011 füllten 381 Personen den Erstfragebogen aus. Von diesen wurden 36 Personen aufgrund zu starker psychischer Belastung $(\mathrm{n}=20)$ oder anderer Ausschlusskriterien (bestehende Schwangerschaft, bestehende Psychotherapie, lebten nicht in einem deutschsprachigen Land, Alter unter 18 Jahre, Kind nach der Geburt verloren; $\mathrm{n}=16$ ) von der Studie ausgeschlossen. Weitere 27 Interessenten haben bereits vor Beginn aus unbekannten Gründen auf eine Teilnahme verzichtet, waren nicht mehr erreichbar oder wurden vor Beginn der Behandlung erneut schwanger. Die 228 Teilnehmenden, die die Teilnahmebedingungen erfüllten und eine Einverständniserklärung unterschrieben hatten, wurden per Randomisierung der BG $(\mathrm{n}=115)$ oder der WL $(\mathrm{n}=113)$ zufällig zugeordnet. Die beiden Gruppen unterschieden sich hinsichtlich soziodemografischer und schwangerschaftsbezogener Daten nicht voneinander.

Die 115 zufällig der BG zugelosten Teilnehmenden waren im Durchschnitt 33,8 Jahre alt (Standardabweichung (SD) = 5,2; Range: 26-47 Jahre), zu 93,9\% Frauen und zu 94,8\% in fester Partnerschaft. 82,9\% hatten als höchsten Schulabschluss das Abitur. Seit dem Kindsverlust waren im Durchschnitt 8,8 Monate (SD = 19,4, Range: 0,5-144 Monate) vergangen. Die meisten Befragten erlebten einen Frühabort (47\%), 18\% einen Spätabort, 13\% einen medizinisch indizierten Schwangerschaftsabbruch und $22 \%$ eine Totgeburt.

16 der 115 Teilnehmenden schieden im Verlauf der Behandlung aus verschiedenen Gründen aus $(n=9$ nicht mehr erreichbar; $n=6$ stellten während der Behandlung fest, dass eine Schreibtherapie für sie nicht der richtige Ansatz sei; $n=1$ beendete die Behandlung aufgrund eines Todesfalls in der Familie). Die verbliebenen 99 Teilnehmenden (86,1\%) der BG beendeten die Behandlung und nahmen an der Post-Messung teil. Sie bildeten die Grundlage für die Berechnungen in der vorliegenden Studie. Abbrecher und Nichtabbrecher unterschieden sich hinsichtlich der demografischen und schwangerschaftsbezogenen Daten mit einer Ausnahme nicht voneinander. Die Abbrecher waren insgesamt jünger $(\mathrm{t}(97)=-2,272$, $\mathrm{p}$ =0,025). Hinsichtlich der gemessenen Symptome zeigten sich keine signifikanten Unterschiede zum Zeitpunkt der Baseline-Messung. Auch in Bezug auf die soziale Unterstützung ergaben sich keine Unterschiede zwischen Abbrechern und Nichtabbrechern der Behandlungsgruppe.

\section{Änderung der sozialen Unterstützung nach der internetbasierten Intervention}

Die durchschnittlichen Werte für die einzelnen Subskalen der sozialen Unterstützung zu den beiden Messzeitpunkten sind in Tabelle $1 \mathrm{zu}$ finden. Sie reichen zum Zeitpunkt der Baseline-Messung von 2,5 (Suche nach Unterstützung) bis 3,36 (wahrgenommene Unterstützung) und ändern sich im Verlauf vom Zeitpunkt der Baseline-Messung zum Zeitpunkt des Abschlusses der Behandlung alle signifikant. Während sich das Bedürfnis nach sozialer Unterstützung zum Ende der Behandlung reduziert hat, nahmen die wahrgenommene, die erhaltene und die Suche nach Unterstützung signifikant zu. Die Prä-/ Post-Effektstärken (Cohens d) lagen zwischen 0,19 und 0,24. 
Tab. 1. Unterschiede der Mittelwerte zwischen den Dimensionen der sozialen Unterstützung vor und nach internetbasierter Intervention

\begin{tabular}{|c|c|c|c|c|c|c|}
\hline & \multicolumn{2}{|c|}{ Prä } & \multicolumn{2}{|c|}{ Post } & \multirow[b]{2}{*}{$\mathrm{p}$} & \multirow[b]{2}{*}{ d } \\
\hline & M & SD & M & SD & & \\
\hline Wahrgenommene Unterstützung & 3,36 & 0,61 & 3,50 & 0,56 & $0,007 * *$ & 0,24 \\
\hline Erhaltene Unterstützung & 3,06 & 0,69 & 3,19 & 0,65 & $0,036^{*}$ & 0,19 \\
\hline Bedürfnis nach Unterstützung & 2,87 & 0,57 & 2,75 & 0,53 & $0,017^{*}$ & 0,22 \\
\hline Suche nach Unterstützung & 2,50 & 0,61 & 2,63 & 0,66 & $0,022 *$ & 0,20 \\
\hline
\end{tabular}

Tab. 2. Korrelationen der Dimensionen sozialer Unterstützung mit den Skalen psychischer Belastung vor Behandlungsbeginn

\begin{tabular}{|c|c|c|c|c|c|c|c|}
\hline \multirow[b]{2}{*}{ BSSS (Prä-Messung) } & \multicolumn{3}{|l|}{ BSI } & \multirow{2}{*}{$\begin{array}{l}\text { ICG } \\
\text { Prolongierte } \\
\text { Trauer }\end{array}$} & \multicolumn{3}{|l|}{ IES-R } \\
\hline & Depressivität & Ängstlichkeit & GSI & & Intrusion & Vermeidung & Übererregung \\
\hline Wahrgenommene Unterstützung & $-0,427 * *$ & $-0,344 * *$ & $-0,454 * *$ & $-0,361 * *$ & $-0,054$ & $-0,152$ & $-0,209 *$ \\
\hline Erhaltene Unterstützung & $-0,282 * *$ & $-0,197$ & $-0,255^{*}$ & $-0,206^{*}$ & 0,046 & $-0,120$ & $-0,028$ \\
\hline Bedürfnis nach Unterstützung & 0,067 & 0 & 0,056 & 0,082 & $0,204^{*}$ & $-0,126$ & 0,081 \\
\hline $\begin{array}{l}\text { BSSS = Berliner Social Support Sk } \\
\text { IES-R = Impact of Event Scale-R } \\
* \mathrm{p}<0,05 . \\
* * \mathrm{p}<0,01 .\end{array}$ & $\begin{array}{l}\text { n; BSI = Brief } \\
\text { ed. }\end{array}$ & ptom Inventc & $\mathrm{GSI}=\mathrm{G}$ & I Severity Inc & $\mathrm{CG}=\mathrm{Inv}$ & ry of Compli & ted Grief; \\
\hline
\end{tabular}

\section{Zusammenhang zwischen sozialer Unterstützung und}

Symptomatik vor der Behandlung

Tabelle 2 gibt eine Übersicht über die Korrelationen zwischen den verschiedenen Dimensionen der sozialen Unterstützung und den Werten der eingesetzten Messinstrumente hinsichtlich der psychischen Belastung vor Behandlungsbeginn (Prä-Messung). Während eine höhere «wahrgenommene soziale Unterstützung» mit einer geringeren Symptombelastung einherging, also mit allen erfassten Symptomen außer den Subskalen «Intrusion» und «Vermeidung» des IES-R signifikant negativ korrelierte, ergaben sich für die «erhaltene soziale Unterstützung» keinerlei signifikante Korrelationen mit dem IES-R und auch nicht mit der Subskala «Ängstlichkeit» des BSI. Das Bedürfnis nach Unterstützung wurde mit zunehmender Intrusion größer, was sich in einer positiven Korrelation dieser beiden Variablen zeigte. Im Gegensatz dazu schienen Teilnehmer mit stärkerer Depressivität weniger nach Unterstützung zu suchen, was eine negative Korrelation der Depressivität mit der Suche nach Unterstützung ergab.

\section{Zusammenhang zwischen sozialer Unterstützung und Behandlungsergebnis}

Tabelle 3 gibt eine Übersicht über die Korrelationen zwischen den verschiedenen Dimensionen der sozialen Unterstützung und den Post-Werten der eingesetzten Messinstrumente bzw. deren RGS. Sowohl für die Post-Werte der Symptome als auch für die durch den RGS erfassten Prä-/Post-
Veränderungen ergaben sich in der Tendenz negative Korrelationen mit den verschiedenen Aspekten sozialer Unterstützung. Dies bedeutet, dass höhere wahrgenommene und erhaltene soziale Unterstützung sowie ein höheres Bedürfnis und eine vermehrte Suche nach Unterstützung sowohl mit geringerer Symptomstärke zum Zeitpunkt nach der Behandlung als auch mit einer stärkeren Prä-/Post-Veränderung der Symptomatik einhergingen. Während für die «wahrgenommene soziale Unterstützung» und die Post-Werte der eingesetzten Messinstrumente, mit Ausnahme der Skalen «Intrusion» und «Übererregung» des IES-R, signifikante negative Korrelationen gefunden werden konnten, zeigten sich diese für die entsprechenden RGS nur noch in der Subskala «Übererregung» des IES-R. Im Gegensatz dazu ergaben sich für die BSSS «erhaltene Unterstützung» mit den RGS ähnliche Korrelationen wie mit den Post-Werten. Während letztere mit der Ausnahme des ICG alle signifikant negativ mit der «erhaltenen sozialen Unterstützung» korrelierten, ergaben sich für die RGS mit der Subskala «Ängstlichkeit» des BSI und der Skala «Vermeidung» des IES-R 2 weitere Ausnahmen. Diese beiden Skalen zeigten bei den Post-Werten auch als einzige keine signifikanten Korrelationen mit der BSSS «Suche nach Unterstützung». Bei den entsprechenden RGS ergab sich zusätzlich keine signifikante Korrelation mit der Subskala «Depressivität».

Im Gegensatz zu den 3 bereits genannten Skalen des BSS ergaben sich für die Subskala «Bedürfnis nach Unterstüt- 
Tab. 3. Korrelationen der Dimensionen sozialer Unterstützung mit den Skalen psychischer Belastung nach Behandlungsbeginn (Post-Werte) bzw. deren RGS

\begin{tabular}{|c|c|c|c|c|c|c|c|}
\hline \multirow[b]{2}{*}{ BSSS } & \multicolumn{3}{|l|}{ BSI } & \multirow{2}{*}{$\begin{array}{l}\text { ICG } \\
\text { Prolongierte } \\
\text { Trauer }\end{array}$} & \multicolumn{3}{|l|}{ IES-R } \\
\hline & Depresivität & Ängstlichkeit & GSI & & Intrusion & Vermeidung & Übererregung \\
\hline \multicolumn{8}{|c|}{ Wahrgenommene Unterstützung } \\
\hline Post & $-0,325 * *$ & $-0,276^{* *}$ & $-0,398 * *$ & $-0,206^{*}$ & $-0,158$ & 0,086 & $-0,304 * *$ \\
\hline RGS & $-0,175$ & $-0,148$ & $-0,198$ & $-0,060$ & $-0,150$ & $-0,009$ & $-0,233^{*}$ \\
\hline \multicolumn{8}{|c|}{ Erhaltene Unterstützung } \\
\hline Post & $-0,370 * *$ & $-0,209 *$ & $-0,346 * *$ & $-0,194$ & $-0,211^{*}$ & $-0,212 *$ & $-0,308 * *$ \\
\hline RGS & $-0,288 * *$ & $-0,150$ & $-0,262 * *$ & $-0,071$ & $-0,256^{*}$ & $-0,175$ & $-0,333 * *$ \\
\hline \multicolumn{8}{|c|}{ Bedürfnis nach Unterstützung } \\
\hline Post & $-0,101$ & $-0,113$ & $-0,084$ & $-0,022$ & $-0,137$ & $-0,132$ & $-0,110$ \\
\hline RGS & $-0,127$ & $-0,103$ & $-0,111$ & $-0,109$ & $-0,251^{*}$ & $-0,078$ & $-0,167$ \\
\hline \multicolumn{8}{|c|}{ Suche nach Unterstützung } \\
\hline Post & $-0,267 * *$ & $-0,083$ & $-0,249 *$ & $-0,311 * *$ & $-0,346 * *$ & $-0,151$ & $-0,321 * *$ \\
\hline RGS & $-0,193$ & $-0,041$ & $-0,160$ & $-0,262 * *$ & $-0,329 * *$ & $-0,094$ & $-0,264 * *$ \\
\hline \multicolumn{8}{|c|}{$\begin{array}{l}\text { BSSS = Berliner Social Support Skalen; BSI = Brief Symptom Inventory; GSI = Global Severity Index; ICG = Inventory of Complicated Grief; } \\
\text { IES-R = Impact of Event Scale-Revised; RGS = Residual Gain Score. } \\
* \mathrm{p}<0,05 \\
* * \mathrm{p}<0,01\end{array}$} \\
\hline
\end{tabular}

zung» keine signifikanten Zusammenhänge mit den PostWerten der Ergebnisfragebögen. Mit einer Ausnahme, der Skala «Intrusion» des IES-R, blieben die signifikanten Korrelationen auch für die RGS der einzelnen Subskalen aus.

\section{Diskussion}

Ziel der vorliegenden Studie war es, erstmalig den Zusammenhang verschiedener Dimensionen sozialer Unterstützung mit Aspekten psychischer Belastung nach einem Kindsverlust vor und nach einer internetbasierten Intervention zu untersuchen und den Zusammenhang zwischen der sozialen Unterstützung und der Veränderung der psychischen Belastung nach Ende der Intervention zu fokussieren.

Die Einschätzung der Studienteilnehmer zur sozialen Unterstützung in den verschiedenen Dimensionen unterscheidet sich nach Ende der Intervention signifikant von der Wahrnehmung zu Behandlungsbeginn. Die in diesem Zusammenhang ermittelten Effektstärken sind jedoch als gering einzustufen [Cohen, 1988]. Da die Intervention, an der die Teilnehmer partizipierten, nicht direkt eine Veränderung der Dimensionen der sozialen Unterstützung adressierte, ist dieses Ergebnis nicht überraschend. Während das Bedürfnis nach Unterstützung sank, nahm die wahrgenommene und erhaltene Unterstützung sowie die Suche nach Unterstützung zu, was einen Hinweis darauf gibt, dass die Betroffenen sich einerseits besser unterstützt fühlten als noch zu Beginn der Behandlung und andererseits aktiver nach Hilfe suchten. Letzteres wurde vermutlich vor allem durch die 3. Phase der Internetintervention - das Social Sharing - angeregt, in der das Aktivieren des ei- genen sozialen Netzwerks geplant und so die soziale Unterstützung verbessert werden sollte. Die aktive Suche nach Unterstützung beugt nachweislich einer gefühlten Isolation vor, die häufig von Eltern berichtet wird, die ihr Kind verloren haben [Kavanaugh et al., 2004]. Dies hat Implikationen für das Versorgungs- und Gesundheitssystem. Beratende Unterstützung vonseiten der Kliniken oder Beratungsstellen, Aufklärung über die schützende Wirkung sozialer Netzwerke, von entsprechenden Institutionen eingerichtete Möglichkeiten, an die verstorbenen Kinder zu erinnern, oder Gruppenangebote zum Austausch und Erlernen von Bewältigungsstrategien sind für die Trauerarbeit vielfach als hilfreich wahrgenommen und somit als Schutz vor längerfristig bestehender verlustbezogener Psychopathologie beschrieben worden [Forrest et al., 1982; Conway and Russell, 2000; Cote-Arsenault and Freije, 2004].

Dass sich die trauernden Eltern nach der Behandlung besser unterstützt fühlten, ist möglicherweise auf die Beziehung zurückzuführen, die sich während der Behandlung zwischen der teilnehmenden Person und der betreuenden Therapeutin entwickelte. Die therapeutische Beziehung wurde auch in anderen internetbasierten Behandlungsprogrammen als sehr positiv eingeschätzt [Klasen et al., 2013; Dölemeyer et al., 2013a]. Möglicherweise nehmen die Personen den Therapeuten als enge Bezugsperson wahr oder fühlen sich durch die Therapie sozial unterstützt. So empfand beispielsweise in anderen internetbasierten Interventionen ein Großteil der Studienteilnehmer den Kontakt zum Therapeuten als sehr angenehm (88\%) [Lange et al., 2003] bzw. als sehr persönlich (86\%) [Knaevelsrud und Maercker, 2007].

Für die meisten Symptombereiche vor Behandlungsbeginn bestätigt der inverse Zusammenhang zwischen wahrgenomme- 
ner sozialer Unterstützung und psychischer Belastung den gut belegten Befund, dass Eltern, die während der Schwangerschaft ein Kind verloren haben, weniger psychisch belastet sind, wenn sie sich von nahestehenden Bezugspersonen wie Angehörigen und Freunden unterstützt fühlen [Kuse-Isingschulte et al., 1996; Janssen et al., 1997; Kavanaugh et al., 2004; Kersting und Wagner, 2012; Sutan und Miskam, 2012]. Zudem gingen mit mehr erhaltener Unterstützung in der spezifischen Situation des Kindsverlusts signifikant weniger Symptome der Depressivität und prolongierten Trauer einher, was die Befunde zur prolongierten Trauer vom Einfluss der wahrgenommenen Unterstützung auf die erhaltene Unterstützung ausweitet. Personen, die mehr Intrusionen in Bezug auf das Verlusterlebnis hatten, gaben auch ein höheres Bedürfnis nach Unterstützung an. Hohe Werte der Depressivität sind negativ mit der Suche nach Unterstützung assoziiert, was dem Krankheitsbild der depressiven Störung und im Speziellen dem Symptom der Antriebsarmut entspricht [Hautzinger, 2009].

Auch nach der internetbasierten Intervention finden wir teilweise inverse signifikante Zusammenhänge zwischen einzelnen Dimensionen der sozialen Unterstützung und Symptomen der psychischen Belastung. Betrachtet man jedoch die RGS, also die messfehlerbereinigten Veränderungen der psychischen Belastung nach der Intervention, bleiben Zusammenhänge zwischen den Belastungsdimensionen «Depressivität», «GSI», «Intrusion» und «Übererregung» mit der erhaltenen Unterstützung bestehen. Dieses Ergebnis zeigt, dass für die beschriebenen Symptomdimensionen eine bessere erhaltene soziale Unterstützung mit einer Verbesserung der Symptome nach Beenden der internetbasierten Intervention einhergeht, was wiederum den abfedernden Effekt der Ressource zeigt. In Bezug auf die Suche nach sozialer Unterstützung blieben die Korrelationen für die RGS mit der prolongierten Trauer, Intrusion und Übererregung bestehen. Je stärker die Suche nach Unterstützung war, umso mehr reduzierten sich diese trauerbezogenen Symptome. Dieser Befund bestätigt bereits vorliegende Ergebnisse [Lasker und Toedter, 1991; Janssen et al., 1997]. Es wird deutlich, dass aktive, an einer Problemlösung orientierte Bewältigungsstrategien protektiv sein können, und dass unterstützungssuchende Aktivitäten bei Betroffenen angeregt werden sollten. Neben Gruppen- und Einzelangeboten, mit denen Kliniken oder Beratungsstellen bzw. andere Institutionen des Gesundheitssystems die Betroffenen unterstützen können, stellen somit auch internetbasierte Behandlungsangebote eine gute Unterstützungsmöglichkeit dar. So konnte nicht nur deren Wirksamkeit gezeigt werden [Kersting et al., 2011a; 2013]; aufgrund der Anonymität des Internets erleichtern sie die Hilfesuche insbesondere Betroffenen, die sich sozial zurückgezogen haben. Einschränkend ist allerdings zu bemerken, dass Fernbehandlungen, ergo auch psychotherapeutische Behandlungen über das Internet, im Rahmen der Regelversorgung in Deutschland derzeit nicht standardmäßig abgedeckt werden dürfen [Almer und Warntjen, 2009], sodass hier eine Weiterentwicklung wünschenswert wäre.
Auch die Kürze des hier vorgestellten 5-wöchigen Programms könnte, im Gegensatz zu psychotherapeutischen Face-to-face-Behandlungen, ein Faktor sein, der die Wahrscheinlichkeit der Inanspruchnahme erhöht. Interessant wäre es zu untersuchen, ob sich die Zusammenhänge zwischen dem Erfolg einer Therapie und den Arten sozialer Unterstützung (in diesem Falle die «erhaltene Unterstützung» und die «Suche nach Unterstützung») auch für die Face-to-face-Therapie feststellen ließen. Weitere Untersuchungen könnten dieser Fragestellung nachgehen.

Einige Limitationen sollten bei der Interpretation der Ergebnisse berücksichtigt werden. Bei den Fragen zur erhaltenen sozialen Unterstützung wurden die Studienteilnehmer gebeten, an die engste Bezugsperson zu denken. Es ist nicht auszuschließen, dass bei dieser Bewertung auch die Unterstützung durch den Therapeuten mit einfließt. In zukünftigen Studien könnte dieser Kritik durch eine klare Benennung der Bezugsperson begegnet werden.

Bei den Teilnehmern handelt es sich um eine hochselektive Stichprobe, die nach strengen Forschungskriterien ausgewählt wurde. So stellt die Behandlung via Internet nicht für jedes Individuum einen adäquaten Behandlungsweg dar, da Begleitsymptome wie z.B. psychotische Symptomatik oder Suizidalität ausgeschlossen werden. Zudem ist der Frauenanteil mit 93,9\% sehr hoch, worin sich möglicherweise die Tatsache widerspiegelt, dass Frauen häufiger psychotherapeutische Hilfe suchen als Männer [Wagner et al., 2012; Dölemeyer et al., 2013a; Kersting et al. 2013]. Aus diesem Grund können mit der vorliegenden Untersuchung keine Aussagen zu möglichen Geschlechterunterschieden getroffen werden.

Es gibt Hinweise darauf, dass sich Frauen nach einer frühen Fehlgeburt mehr unterstützt fühlen als ihre Partner [Conway und Russell, 2000]. Zudem mobilisieren und erhalten Frauen meist mehr soziale Unterstützung als Männer [Matthews et al., 1999] und sind meist zufriedener mit der Unterstützung, die sie erhalten [Antonucci and Akiyama, 1987]. Auch die Wahrnehmung der sozialen Unterstützung als hilfreich oder wenig hilfreich ist stark geschlechterabhängig [Kavanaugh et al., 2004]. Mit Hilfe zukünftiger Studien könnten diese interessanten Geschlechterunterschiede weiter untersucht werden. Eine weitere Besonderheit der Stichprobe stellt das hohe Bildungsniveau der Teilnehmenden dar. Dieses unterstreicht die Ergebnisse anderer Studien, wonach internetbasierte Verfahren eher von gebildeten Personen genutzt werden [Wagner et al., 2006; Knaevelsrud und Maercker, 2007; Dölemeyer et al. 2013b].

Trotz der methodischen Einschränkungen deuten die Ergebnisse darauf hin, dass das hier vorgestellte internetbasierte Behandlungsangebot nach dem pränatalen Verlust eines Kindes eine gute Unterstützungsmöglichkeit darstellt, die aktivierend bei der Suche nach Unterstützung wirken kann. Sowohl die erhaltene soziale Unterstützung als auch die Suche nach sozialer Unterstützung geht mit einer Reduzierung der psychopathologischen Belastung nach dem Kindsverlust einher. 


\section{Disclosure Statement}

Die Autoren erklären hiermit, dass keine Interessenskonflikte vorliegen.

\section{Literatur}

-Almer D, Warntjen D: Psychotherapie und Internet. Psychotherapeut 2009;54:393-396.

Antonucci T, Akiyama H: An examination of sex differences in social support among older men and women. Sex Roles 1987;17:737-749.

-Bergner A, Beyer R, Klapp BF, Rauchfuss M: Pregnancy after early pregnancy loss: a prospective study of anxiety, depressive symptomatology and coping. J Psychosom Obstet Gynaecol 2008;29:105-113.

Bergner A, Beyer R, Klapp BF, Rauchfuss M: Trauer, Bewältigung und subjektive Ursachenzuschreibungen nach Frühaborten: Adaptivität von Verarbeitungsmustern untersucht in einer Langsschnittstudie. Psychother Psych Med 2009;59:57-67.

Berth H, Puschmann AK, Dinkel A, Balck F: Trauma Fehlgeburt - Einflussfaktoren auf das Angsterleben nach dem frühen Verlust eines Kindes. Psychother Psych Med 2009;59:314-320.

Brier N: Anxiety after miscarriage: a review of the empirical literature and implications for clinical practice. Birth 2004;31:138-142.

Brier N: Grief following miscarriage: a comprehensive review of the literature. J Womens Health 2008;17: 451-464.

Carlbring P, Apelstrand M, Sehlin H, Amir N, Rousseau A, Hofmann SG, Andersson G: Internet-delivered attention bias modification training in individuals with social anxiety disorder - a double blind randomized controlled trial. BMC Psychiatry 2012;12:66.

Cohen J: Statistical Power Analysis for the Behavioral Sciences. Hillsdale, Lawrence Erlbaum Associates, 1988.

Conway K: Miscarriage experience and the role of support systems: a pilot study. Br J Med Psychol 1995;68(Pt 3):259-267.

Conway K, Russell G: Couples' grief and experience of support in the aftermath of miscarriage. Br J Med Psychol 2000;73(Pt 4):531-545.

Cote-Arsenault D, Freije MM: Support groups helping women through pregnancies after loss. West J Nurs Res 2004;26:650-670.

-Cuisinier MC, Kuijpers JC, Hoogduin CA, de Graauw $\mathrm{CP}$, Janssen HJ: Miscarriage and stillbirth: time since the loss, grief intensity and satisfaction with care. Eur J Obstet Gynecol Reprod Biol 1993;52:163-168.

Dölemeyer R, Tietjen A, Kersting A, Wagner B: Internet-based interventions for eating disorders in adults: a systematic review. BMC Psychiatry 2013a 13:207.

Dölemeyer R, Klinitzke G, Steinig J, Wagner B, Kersting A: Die therapeutische Beziehung in einem internetbasierten Programm zur Behandlung der Binge-Eating-Störung. Psychiatr Prax 2013b;DOI: 10.1055/s-0033-1343320.

Forrest GC, Standish E, Baum JD: Support after perinatal death: a study of support and counselling after perinatal bereavement. Br Med J (Clin Res Ed) 1982;285:1475-1479.

Franke G: Brief Symptom Inventory (BSI). Göttingen, Beltz, 2000.

- Geller PA, Psaros C, Kornfield SL: Satisfaction with pregnancy loss aftercare: are women getting what they want? Arch Womens Ment Health 2010;13: $111-124$
Gold KJ, Dalton VK, Schwenk TL: Hospital care for parents after perinatal death. Obstet Gynecol 2007; 109:1156-1166.

Hautzinger M: Depression; in Margraf J, Schneider S (eds): Lehrbuch der Verhaltenstherapie. Berlin, Springer, 2009, pp 125-138.

Hobfoll SE: Conservation of resources. A new attempt at conceptualizing stress. Am Psychol 1989;44:513524

Janssen HJ, Cuisinier MC, de Graauw KP, Hoogduin KA: A prospective study of risk factors predicting grief intensity following pregnancy loss. Arch Gen Psychiatry 1997:54:56-61.

Kavanaugh K, Trier D, Korzec M: Social support following perinatal loss. J Fam Nurs 2004;10:70-92.

Kersting A: Peripartale Depressionen und Trauer nach Schwangerschaftsverlusten: Besondere Problemfelder in der Geburtshilfe. Der Nervenarzt 2012;83:1434-1441.

Kersting A, Kroker K, Schlicht S, Baust K, Wagner B: Efficacy of cognitive behavioral internet-based therapy in parents after the loss of a child during pregnancy: pilot data from a randomized controlled trial. Arch Womens Ment Health 2011a;14:465-477.

Kersting A, Brähler E, Glaesmer H, Wagner B: Prevalence of complicated grief in a representative population-based sample. J Affect Disord 2011b;131: 339-343.

Kersting A, Kroker K, Schlicht S, Wagner B: Internetbased treatment after pregnancy loss: concept and case study. J Psychosom Obstet Gynaecol 2011c; 32:72-78.

Kersting A, Dölemeyer R, Steinig J, Walter F, Kroker $\mathrm{K}$, Baust K, Wagner B: Brief internet-based intervention reduces posttraumatic stress and prolonged grief in parents after the loss of a child during pregnancy: a randomized controlled trial. Psychother Psychosom 2013;DOI:10.1159/000348713.

Kersting A, Wagner B: Complicated grief after perinatal loss. Dialogues Clin Neurosci 2012;14:187-194.

Kienle R, Knoll N, Renneberg B: Soziale Ressourcen und Gesundheit: soziale Unterstützung und dyadisches Bewältigen; in Renneberg B, Hammelstein P (eds): Gesundheitspsychologie. Berlin, Springer, 2006, pp 107-122.

Klasen M, Knaevelsrud C, Böttche M: Die therapeutische Beziehung in internetbasierten Therapieverfahren: Ein Überblick. Der Nervenarzt 2013;84: 823-831.

Klauer T, Schwarzer R: Soziale Unterstützung und Depression. Verhaltenstherapie und Verhaltensmedizin 2001;22:333-352.

Knaevelsrud C, Maercker A: Internet-based treatment for PTSD reduces distress and facilitates the development of a strong therapeutic alliance: a randomized controlled clinical trial. BMC Psychiatry 2007; 7:13.

Kuse-Isingschulte MW, Beutel M, Hahlweg BC, Stauber M, Schneider K: Die psychische Verarbeitung einer Totgeburt. Verlauf der Trauerreaktion, Einflussfaktoren, Behandlungszufriedenheit, Betreuungwünsche. Geburtshilfe Frauenheilkd 1996;56:380-389.
Lange A, Rietdijk D, Hudcovicova M, van de Ven JP, Schrieken B, Emmelkamp PM: Interapy: a controlled randomized trial of the standardized treatment of posttraumatic stress through the internet. J Consult Clin Psychol 2003;71:901-909.

Lasker JN, Toedter LJ: Acute versus chronic grief: the case of pregnancy loss. Am J Orthopsychiat 1991; 61:510-522.

Leppert PC, Pahlka BS: Grieving characteristics after spontaneous abortion: a management approach. Obstet Gynecol 1984;64:119-122.

Lok IH, Neugebauer R: Psychological morbidity following miscarriage. Best Pract Res Clin Obstet Gynaecol 2007;21:229-247.

Maercker A, Schützwohl M: Erfassung von psychischen Belastungsfolgen: Die Impact of Event Skala-revidierte Version (IES-R). Diagnostica 1998;44:130-141.

Matthews S, Stansfeld S, Power C: Social support at age 33: the influence of gender, employment status and social class. Soc Sci Med 1999;49:133-142.

McLellan AT, O’Brien CP, Luborsky L, Druley KA, Woody GE: Certain types of substance abuse patients do better in certain kinds of treatments: evidence for patient-program matching. NIDA Res Monogr 1981;34:123-130.

Neugebauer R, Kline J, Markowitz JC, Bleiberg KL, Baxi L, Rosing MA, Levin B, Keith J: Pilot randomized controlled trial of interpersonal counseling for subsyndromal depression following miscarriage. J Clin Psychiatry 2006;67:1299-1304.

Park KO, Wilson MG, Lee MS: Effects of social support at work on depression and organizational productivity. Am J Health Behav 2004;28:444-455.

Prigerson HG, Maciejewski PK, Reynolds CF 3rd, Bierhals AJ, Newsom JT, Fasiczka A, Frank E, Doman J, Miller M: Inventory of Complicated Grief: a scale to measure maladaptive symptoms of loss. Psychiatry Res 1995;59:65-79.

Schulz U, Schwarzer R: Soziale Unterstützung bei der Krankheitsbewältigung: Die Berliner Social Support Skalen (BSSS). Diagnostica 2003;49:73-82.

Steketee G, Chambless DL: Methodological issues in prediction of treatment outcome. Clin Psychol Rev 1992;12:387-400.

Sutan R, Miskam HM: Psychosocial impact of perinatal loss among Muslim women. BMC Womens Health 2012;12:15.

Swanson KM, Chen HT, Graham JC, Wojnar DM, Petras A: Resolution of depression and grief during the first year after miscarriage: a randomized controlled clinical trial of couples-focused interventions. J Womens Health 2009;18:1245-1257.

Toedter LJ, Lasker JN, Janssen HJ: International comparison of studies using the perinatal grief scale: a decade of research on pregnancy loss. Death Stud 2001;25:205-228.

Wagner B, Knaevelsrud C, Maercker A: Internetbased cognitive-behavioral therapy for complicated grief: a randomized controlled trial. Death Stud 2006;30:429-453.

Wagner B, Brand J, Schulz W, Knaevelsrud C: Online working alliance predicts treatment outcome for posttraumatic stress disorder in war-traumatized patients in Iraq. Depress Anxiety 2012;29:646-651. 